\title{
Laparoscopic management of urachal remnants in women affected of pelvic pain
}

\author{
Juan Gilabert-Estelles • Juan Gilabert-Aguilar
}

Received: 2 November 2009 / Accepted: 23 November 2009/Published online: 18 December 2009

(C) Springer-Verlag 2009

\begin{abstract}
Symptomatic urachal cysts are very infrequently encountered in women affected of pelvic pain and uterine myomas. We report on two female patients aged 40 and 49 years, who underwent complete transperitoneal laparoscopic excision of urachal cysts at our unit. The first patient suffered from infertility and presented a uterine myoma, which was treated with laparoscopic myomectomy. The second case referred was menorrhagia and chronic pelvic pain, and she was treated with a total laparoscopic hysterectomy with complete excision of urachal cyst. All two operations had no serious immediate complications and minimal blood loss. Cysts were located at the lower third of the urachus, and no communication was detected between the cyst and the bladder. The bladder apex was reinforced using suturing in both cases, and a postoperative cystoscopy confirmed the integrity of the bladder wall. Patients were discharged uneventfully on the second postoperative day. In conclusion, complete excision of urachal remnants in women affected of pelvic pain is advisable in order to treat the symptoms and can be safely performed by a laparoscopic approach.
\end{abstract}

J. Gilabert-Estelles $(\bowtie) \cdot J$. Gilabert-Aguilar

Unit of Gynecological Endoscopy, Hospital Casa de Salud,

Av. Manuel Candela 41,

46010, 46009 Valencia, Spain

e-mail: juangilaeste@yahoo.es

J. Gilabert-Estelles

Department of Gynecology, University Hospital La Fe,

Valencia, Spain

J. Gilabert-Aguilar

Department of Gynecology, Hospital Arnau de Vilanova,

Valencia, Spain
Keywords Urachus · Laparoscopy · Cyst · Abnormalities . Pelvic pain

\section{Introduction}

The urachus is the embryological remnant of the allantois, which communicates the apex of the bladder with the umbilicus and usually becomes obliterated shortly after birth. Urachal cysts account for one third of urachal obliteration defects with an observed incidence of $1 / 5,000$ live borns $[1,2]$. Even though males are affected three times more than females [3], gynecologists should be aware of this rare condition, which may be encountered while managing women affected of pelvic pain and midline cysts. Cysts of the urachus rarely become symptomatic and may be encountered often during a surgical endoscopy exploration. Complications of these abnormalities have been described, including infection [4], formation of urachal calculi [5], and the potential risk for a malignant change of the, otherwise, benign urachal remnant $[6,7]$. Symptoms of urachal cysts at adult age may be unspecific and include lower abdominal pain, irritative voiding symptoms, periumbilical pain, or palpable abdominal masses [2]. A number of gynecological pathologies, such as ovarian cysts or uterine myomas, may cause these symptoms and might, therefore, hamper an early diagnosis of urachal cysts in menstruating women

A surgical approach is not always necessary in small uncomplicated urachal cysts as symptoms are often related to complications. In women referring pelvic pain, any abdominal abnormalities found during surgery should be corrected, taking into account the fertility status. Therefore, the recommended management of the incidental finding of 
these embryologic defects, in this type of patients, is surgical excision.

Considering the potential complications and the risk of recurrence, excision of the urachal cyst is recommended over drainage [1]. Traditional approach includes the resection of the urachus by open surgery, but an increasing number of cases have been reported detailing minimally invasive approaches to urachal cysts [8-12].

Our experience in the management of urachal cysts in two women affected of pelvic pain and uterine myomas supports that laparoscopy offers a safe and effective management of both gynecological and urachal pathology.

\section{Case 1}

A 49-year-old Caucasian multigravid woman was referred to our endoscopy unit with a 2-year history of hypermenorrea and lower abdominal pain that increased during menstruation. The patient had a good physical status and did not have any previous surgery. Gynecological examination revealed a tenth week myomatous uterus. Vaginal ultrasound showed multiple intramural myomas with normal adnexa and a 6-cm midline unilocular simple cyst that was located next to the uterine fundus. A computed tomography revealed a $61 \times 48 \mathrm{~mm}$ low density image located at midline cranial to the bladder apex, suggesting the diagnosis of urachal cyst. The preoperative laboratory tests were normal. An informed consent was obtained, and risks and benefits of removing the urachal remnant were discussed. A total laparoscopic hysterectomy with bilateral salpingo-oophorectomy (BSO), and transperitoneal excision of the urachal cyst was planned.

Laparoscopy was performed under general endotracheal anesthesia with the patient in a dorsal lithotomy position. After induction of pneumoperitoneum with a veress needle, an 11-mm trocar was inserted $3 \mathrm{~cm}$ over the umbilicus under direct vision for the $0^{\circ}$ laparoscope (Ternamian endotip, Karl Storz, Germany). A 6-cm preperitoneal cystic mass consistent with a cyst of the lower urachus was detected (Fig. 1). Three accessory 5-mm trocars were placed, one at midline $3 \mathrm{~cm}$ infraumbilically and the other two, laterally to it. First, a standard total laparoscopic hysterectomy with BSO was performed; otherwise, a possible bleeding of the anterior peritoneum while performing the excision of the urachal cyst could have impaired the operative field. The cystoscopy performed discarded the patency of any communication between the cyst and the bladder apex. Then, $200 \mathrm{~cm}^{3}$ of saline serum with methylene blue were left in the bladder in order to facilitate further dissection. Peritoneum was sectioned cranially to the cyst and blunt dissection proceeded laterally. Bipolar courant was used for sealing and cold

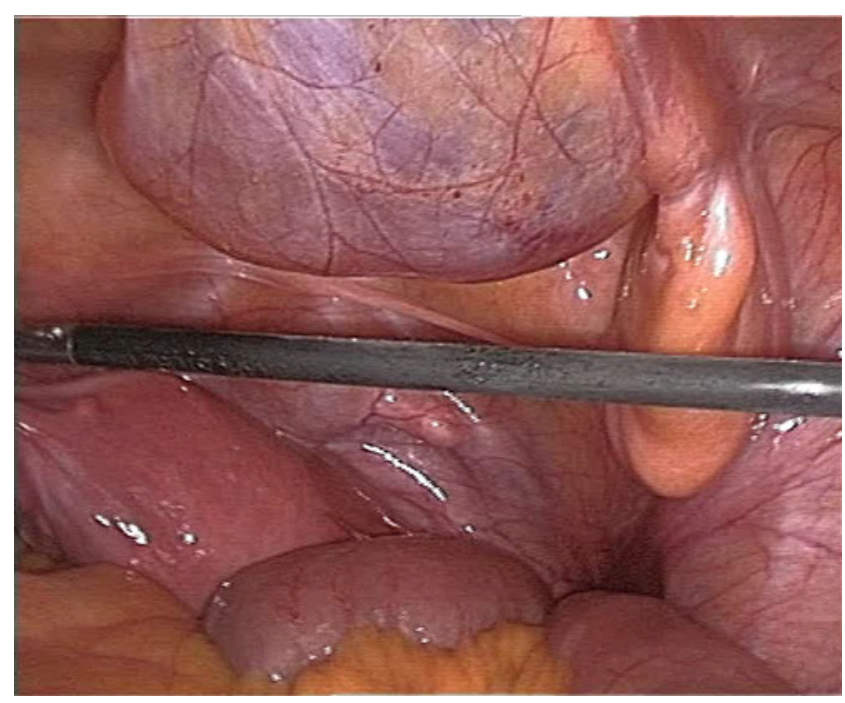

Fig. 1 Laparoscopic view of urachal cyst (Case 1)

scissors allowed a complete enucleation of the cyst, including the urachal tract located cephalad. A wall defect comprising the muscular and serosa layers of the bladder was observed but without any leakage. The bladder was sutured transversally with an interrupted intracorporeal polyglactine 3-0 suture (Fig. 2). The specimen was removed vaginally and extracorporeal polyglactine 0 sutures were used to close the vaginal cuff.

\section{Case 2}

A 40-year-old nulligravid Hispanic woman consulted with the diagnosis of uterine leiomyoma. This patient referred a 2-year history of infertility with no other known additional etiologic factors. She had no medical pathological con-

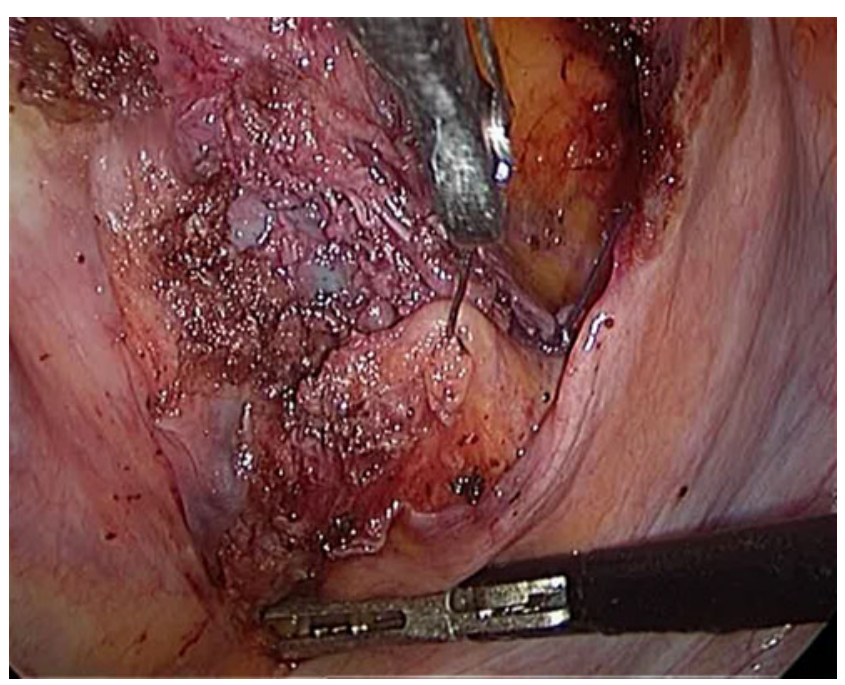

Fig. 2 Reinforcement of the bladder wall with extracorporeal suturing (Case 1) 
ditions except from a mild obesity. Gynecological ultrasound detected a posterior $60 \times 58 \mathrm{~mm}$ intramural myoma, which did not distort the endometrial cavity and ovaries appeared to be normal. A laparoscopic approach to perform a myomectomy and assess tubal patency was planned. Laparoscopic access was performed using an umbilical visual port insertion with a ternamian trocar. The diagnosis of a posterior 6-cm intramural myoma was confirmed. In addition, the examination of the abdominal cavity showed a $3 \times 2 \mathrm{~cm}$ cystic mass subperitoneally located at midline in the anterior abdominal wall (Fig. 3) and an intraoperative cystoscopy ruled out the presence of intraluminal alterations of the bladder. At this point, a small uncomplicated urachal cyst was highly suspected. Given the fact that myomectomy requires the placement of a $12-\mathrm{mm}$ suprapubic trocar for morcellation, we decided to excise the cystic lesion previously. Two lateral $5 \mathrm{~mm}$ trocars were used in order to remove the lesion with a similar procedure to the previous case, after having distended the bladder with $200 \mathrm{ml}$ of saline serum. A polydioxanone endoloop was used in order to seal the urachus cranially to the bladder dome. Once lower resection was completed, urachus was removed cranially to the cyst, including the parietal peritoneum. At the end of the procedure, the bladder was once again distended to check for leaks. Myomectomy was performed uneventfully, and the specimen was extracted after drainage inside an endoscopic bag through the suprapubic trocar.

The total operating time was $120 \mathrm{~min}$ in case 1 and $100 \mathrm{~min}$ in case 2. Neither intraoperative nor postoperative complications occurred. A bladder catheter was maintained for $24 \mathrm{~h}$ in order to avoid bladder distension. Both patients were discharged uneventfully within 2 days after surgery. The excised specimens were sent for histopathological

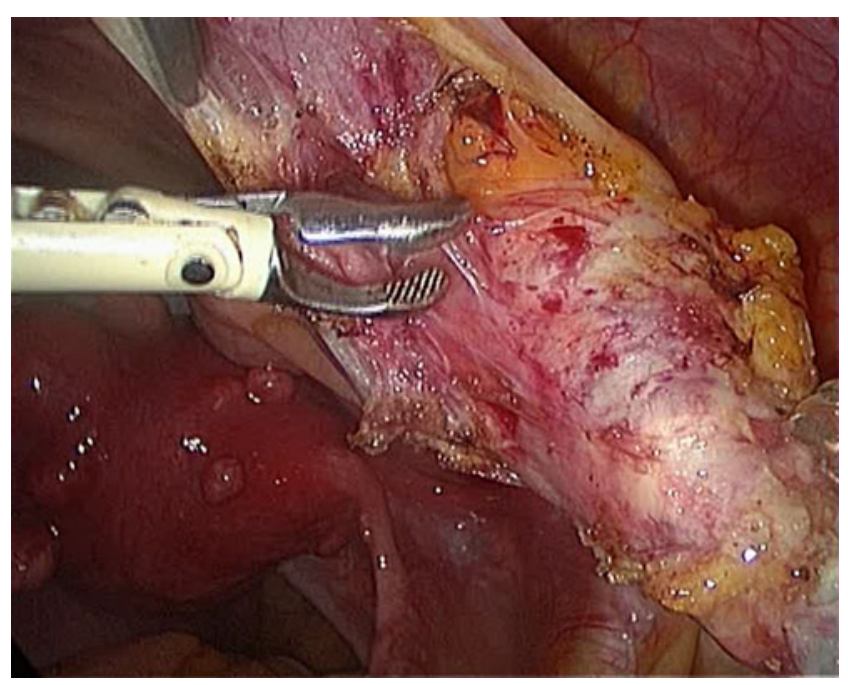

Fig. 3 Opening of the Retzius space cranially to the urachal cyst (Case 2) assessment, and the diagnosis of benign urachal remnants was confirmed.

Currently, both patients remain dry and have not experienced recurrent symptoms with a follow-up period of 8 and 27 months, respectively.

\section{Discussion}

Urachal congenital anomalies are secondary to an abnormal involution of the allantois. Bauer and Retik [13] described four types of anomalies according to the portion of this embryonic structure that remains patent. If the urachus remains patent from the bladder to the umbilicus, it forms a urachal fistula. However, when a patent urachus communicates with the umbilicus but not the bladder, this is called urachal-umbilical sinus. On the contrary, a vesicourachal diverticulum defines a communication between the patent urachus and the dome of the bladder. Finally, the fourth and most common type of urachal remnant is a urachal cyst, which results from the accumulation of mucinous and serous secretions in a cavity suspended at any level of the urachus without communication to either the bladder or to the umbilicus. The most relevant complications of urachal cysts include infection with possible rupture in the peritoneal cavity leading to peritonitis [4] and the risk of malignant degeneration. The most accepted treatment for congenital patent urachus is the excision of the urachal tract, including the parietal peritoneum, from the umbilicus to the bladder. Partial resections of patent urachus may recur up to $30 \%$ [14]. Several authors perform a partial colpectomy, including the dome of the bladder to prevent malignant degeneration. However, we did not consider necessary this practice as the estimated risk of malignancy is lower than $0.1 \%$ [15] and other authors have reported favorable outcomes without resection of the bladder cuff in all the cases [16].

Women at their reproductive age present frequently ovarian cysts, most of them functional and which do not require surgery. The urachal cysts are frequently located at the lower portion of the urachus, and differential diagnosis is mandatory with other adnexal pathology. Gynecological ultrasound is usually performed vaginally with previous voiding of the bladder. Therefore, low urachal cysts are encountered at midline near to the uterine fundus, quite similar to ovarian cysts, and might lead to misdiagnosis of the urachal abnormality. A useful tip consists in filling the bladder, a maneuver which cranially displaces the cyst and facilitates its correct diagnosis [17].

Symptomatic urachal remnants have been traditionally managed by midline infraumbilical incision. Open approach is usually associated with long recovery periods and negative cosmetic results. Neufang et al. reported the 
first laparoscopic excision of a urachal fistula in 1992 [18]. In 1993, Trondsen described a laparoscopic approach in a patient affected of urachal sinus [12], being the first laparoscopic excisions of a urachal cyst reported by Siegel et al. [19] and Jorion [20] in 1994. Since then, several case reports have described successful laparoscopic managements of urachal cysts in different clinical conditions $[9,21$, 22]. There have also been short series describing the laparoscopic management of urachal remnants with a complete excision and a reduced morbidity [6, 8, 23, 24]. Cutting et al. reported one recurrence in five patients treated by laparoscopy due to a lack of resection of the subcutaneous part of the urachus below the umbilicus in an obese patient [11]. Based on their comparative study between the laparoscopic and the open approach to urachal remnants, Okegawa et al. stressed the clinical advantages of minimal invasive surgery [25].

Different techniques have been described for the surgical management of urachal remnants. Transperitoneal technique allows the examination of the abdominal cavity both to rule out other entities and to treat concomitant findings. This approach was mandatory in our cases, as treatment of uterine leiomyomas was the main indication for surgery and was also preferred by Takeda et al., who excised an ovarian dermoid cyst and a urachal cyst found at preoperative examination [26]. LePicard et al. exploited the unique preperitoneal localization of the urachal cyst at the Retzius space to describe an extraperitoneal approach, with an easy blunt dissection and a very selective use of electrocoagulation, which remains a feasible option in cases of isolated urachal pathology [27]. Umbilical access offers good cosmetic results but may reduce the operative field and impair a proper instrument triangulation. In keeping with other authors, we have found the supraumbilical port to be very useful $[11,23]$ and unhampered of limitations to perform a concomitant hysterectomy.

In conclusion, the present report underlines that the laparoscopic excision of urachal remnants in women affected of benign gynecological pathology is a safe and reliable technique. A urachal cyst in the presence of pelvic pain should be removed as soon as an accurate diagnosis has been achieved, even though etiologic relationship is not confirmed.

Conflict of interest There is no actual or potential conflict of interest in relation to this article.

\section{References}

1. Blichert-Toft M, Nielsen OV (1971) Diseases of the urachus simulating intra abdominal disorders. Am J Surg 122:123-128
2. Goldman IL, Caldamone AA, Gauderer M et al (1988) Infected urachal cyst: a review of 10 cases. J Urol 140:375-378

3. Bunch PT, Kline-Fath BM, ImhoV SC, Calvo-Garcia MA, Crombleholme TM, Donnelly LF (2006) Allantoic cyst: a prenatal clue to patent urachus. Pediatr Radiol 36:1090-1095

4. Macneily AE, Koleilat N, Kiriuluta HG et al (1992) Urachal abscesses: protean manifestations, their recognition and management. Urology 40:530

5. Seo IY, Han DY, Oh SJ, Rim JS (2008) Laparoscopic excision of a urachal cyst containing large stones in an adult. Yonsei Med J 49:869-871

6. Cadeddu J, Boyle K, Fabrizio M et al (2000) Laparoscopic management of urachal cysts in adulthood. J Urol 164:15361539

7. Navarrete S, Sanchez-Ismayel A, Sanchez-Salas RE et al (2005) Treatment of urachal anomalies: a minimally invasive surgery technique. JSLS 9:422-425

8. Fahlenkamp D, Schonberger B, Lindeke A, Loening S (1995) Laparoscopic excision of the sinusoidal remnants of the urachus in a 3-year-old boy. Br J Urol 76:135-137

9. Stone NN, Garden R, Weber H (1995) Laparoscopic excision of a urachal cyst. Urology 45:161-164

10. Linos D, Mitropolus F, Patoulis J, Psomas M, Parasyris V (1997) Laparoscopic removal of urachal sinus. J Laparoendosc Adv Surg Tech 7:135-138

11. Cutting CW, Hindley RG, Poulsen J (2005) Laparoscopic management of complicated urachal remnants. BJU Int 966:1417-1421

12. Trondsen E, Reiertsen O, Rosseland AR (1993) Laparoscopic excision of a urachal sinus. Eur J Surg 159:127-128

13. Bauer SB, Retik AB (1978) Urachal abnormalities and related umbilical disorders. Urol Clin North Am 5:195-211

14. Nix J, Menville J, Albert M, Wendt D (1958) Congenital patent urachus. J Urol 79:264-273

15. Pantuck A, Vates T, Javidian P, Weiss R (1997) Urachal adenocarcinoma. Can J Urol 4:450-452

16. Mesrobian HG, Zacharias A, Balcom AH, Cohen RD (1997) Ten years of experience with isolated urachal anomalies in children. $\mathrm{J}$ Urol 158:1316-1318

17. Robert Y, Hennquin C, Chaillet D (1996) Urachal remnants: sonographic assessment. J Clin Ultrasound 24:339

18. Neufang T, Ludtke FE, Lepsien G (1992) Laparoscopic excision of a urachal fistula-new therapy for a rare disorder. Minim Invasive Ther 1:245-249

19. Siegel JF, Winfield H, Valderrama E, Smith A (1994) Laparoscopic excision of urachal cyst. J Urol 151:1631-1633

20. Jorion JL (1994) Laparoscopic removal of urachal cyst. J Urol 151:1006

21. Yamada T, Okamoto Y, Kasamatsu H, Mori H (2001) Laparoscopic assisted removal of a large urachal cyst. J Am Assoc Gynecol Laparosc 8:159-160

22. Turial S, Hueckstaedt T, Schier F, Fahlenkamp D (2007) Laparoscopic treatment of urachal remnants in children. J Urol 177:1864-1866

23. Khurana S, Borzi PA (2002) Laparoscopic management of complicated urachal disease in children. J Urol 168:1526-1528

24. Nakagawa K (2002) Laparoscopic excision of urachal remnant. JJCS 27:884-888

25. Okegawa T, Odagane A, Nutahara K, Higashihara E (2006) Laparoscopic management of urachal remnants in adulthood. Int $\mathrm{J}$ Urol 13:1466-1469

26. Takeda A, Manabe S, Mitsui T, Nakamura H (2005) Laparoscopic excision of urachal cyst found at preoperative examination for ovarian dermoid cyst. Gynecol Surg 3:1

27. LePicard P, Carion G (2002) Extraperitoneal laparoscopic treatment of urachal cyst. J Chir 139:286-287 